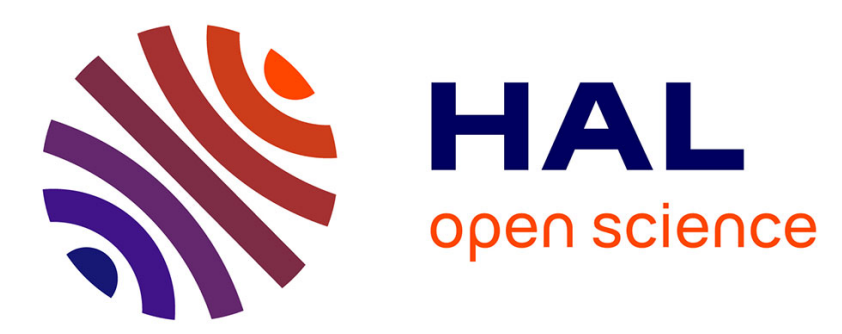

\title{
Choisir ses horaires et en payer le prix. Les horaires flexibles et la conciliation travail-famille des femmes salariées
}

Gregor Bouville

\section{To cite this version:}

Gregor Bouville. Choisir ses horaires et en payer le prix. Les horaires flexibles et la conciliation travailfamille des femmes salariées. @GRH, 2019, 31 (2), pp.9. 10.3917/grh.192.0009 . hal-03227072

\section{HAL Id: hal-03227072 \\ https://hal.science/hal-03227072}

Submitted on 19 May 2021

HAL is a multi-disciplinary open access archive for the deposit and dissemination of scientific research documents, whether they are published or not. The documents may come from teaching and research institutions in France or abroad, or from public or private research centers.
L'archive ouverte pluridisciplinaire HAL, est destinée au dépôt et à la diffusion de documents scientifiques de niveau recherche, publiés ou non, émanant des établissements d'enseignement et de recherche français ou étrangers, des laboratoires publics ou privés. 


\title{
Choisir ses horaires et en payer le prix. Les horaires flexibles et la conciliation travail-famille des femmes salariées.
}

\author{
Gregor Bouville \\ Université Paris-Dauphine, PSL Research University \\ CNRS, UMR [7088], DRM, [Management et Organisation] \\ 75016 PARIS, France.
}

Pour citer : Bouville, G. (2019). Choisir ses horaires et en payer le prix. Les horaires flexibles et la

conciliation travail-famille des femmes salariées. @GRH, $n^{\circ} 31$ (2), 9. doi:10.3917/grh.192.0009.

\section{Résumé}

Dans cet article, nous nous intéressons aux relations entre la flexibilité des horaires et la conciliation travail-famille en distinguant à la fois les hommes et les femmes salariés, les cadres et les non-cadres à travers l'analyse des données $(N=9555)$ d'un panel constitué à partir de la fusion des enquêtes nationales Conditions de travail 2013 et Conditions de travailRPS 2016. Les horaires flexibles améliorent la conciliation travail-famille des femmes noncadres. A l'inverse, nous montrons que loin d'améliorer la conciliation travail-famille des femmes cadres, les horaires flexibles la dégradent. Nous nous intéressons ensuite aux contreparties en termes de gestion du travail mises en place pour les salariés bénéficiant de ces horaires flexibles. Nous montrons alors que, pour les femmes salariées, l'autonomie dans l'organisation de leur temps de travail a pour contrepartie une moindre progression de carrière ainsi que l'exposition à un ensemble de pratiques de gestion du travail néo-managériales, rattaché au courant du High Performance Work System. Nous concluons que l'autonomie concédée au salarié par l'employeur dans l'organisation de son temps de travail a pour contrepartie un contrôle indirect plus fort exercé via l'imposition d'une évaluation régulière, d'une direction par objectifs et d'une rémunération à la performance. Ce contrôle est particulièrement fort pour les femmes cadres.

Mots clés : horaires flexibles, conciliation vie privée-vie professionnelle, genre au travail, bien-être au travail, pratiques de GRH à haute performance (High Performance Work System), organisation et conditions de travail 


\section{Abstract}

In this article, we are interested in the relationships between flexible working hours and worklife balance, distinguishing between men and women employees, managers and non-managers through data analysis (total $N=9555$ ) of a panel formed from the merger of the Working Conditions 2013 and Working Conditions-Psychosocial Risks 2016 national surveys. Flexible working schedules improve the work-family balance of women non-managers. Conversely, we show that far from improving the work-family balance of women executives, flexible schedules degrade her. We are then interested in the labor management counterparts set up for employees benefiting from these flexible schedules. We then show that, for salaried women, the autonomy in the organization of their working time is offset by a lower career progression and exposure to a set of neo-managerial labor management practices, linked to current of the High-Performance Work System. We conclude that the autonomy granted to the employee by the employer in the organization of her working time is counterbalanced by a stronger indirect control exercised through the imposition of a regular performance appraisals, a management by goal setting and a performance-related pay. This control is particularly strong for women managers.

Key words: flexible work arrangements, schedule control, work-life balance, gender at work, well-being, high performance work systems, organization and working conditions 


\section{Introduction}

La caractéristique toujours actuelle de l'environnement extra-professionnel des femmes est l'inégale répartition entre les hommes et les femmes dans la prise en charge des tâches domestiques et familiales. Les femmes actives sont confrontées à une «double journée » de travail (Bratberg et alii, 2002 ; Champagne et alii, 2015), c'est-à-dire qu'elles cumulent dans une même journée obligations professionnelles et familiales / domestiques. Or de nombreux travaux francophones ont montré la difficulté à articuler travail professionnel et travail domestique pour les femmes (Ballesteros-Leiva et alii, 2016 ; Dodeler et Tremblay, 2014 ; Dumas, 2008 ; Lapeyre et Le Feuvre, 2004 ; Pigeyre, 1999, 2001 ; Tremblay et d'Amours, 2016). En cela, les horaires flexibles peuvent être considérés comme un moyen de mieux organiser le «temps de travail $»^{1}$ (Pierce et Newtsrom, 1980) des femmes et ainsi leur difficulté à concilier vie professionnelle et vie privée. En effet, « dans les pays à faible taux de natalité et à population active vieillissante, les gouvernements ont souvent recours aux politiques publiques pour augmenter les congés personnels et parentaux, ainsi que pour adapter les horaires de travail afin d'encourager les femmes à intégrer et développer le marché du travail et de les aider à concilier vie professionnelle et future responsabilités familiales afin de ne pas décourager les naissances » (Berg et alii, 2004, p. 335).

Par horaires flexibles, nous entendons la possibilité pour le salarié de choisir librement ses heures d'arrivée et de départ du travail ou, du moins, la possibilité de choisir ses heures de départ et d'arrivée au sein de plages horaires mobiles. Deux constats nous incitent à nous intéresser à ce sujet. Tout d'abord, la volonté de la Commission Européenne d'encourager le développement des horaires flexibles perçus comme un moyen de résorber la difficile conciliation entre vie privée et vie professionnelle des salariées (Kerkhofs et alii, 2008). Il nous paraît intéressant d'examiner de plus près la portée et les limites de cette solution, alors même qu'une étude, dans le secteur des grands cabinets d'audit comptable, montre que les horaires flexibles, à l'inverse du but recherché, renforçaient les inégalités entre les hommes et les femmes en accentuant les obstacles à la progression des carrières féminines (Kornberger et alii, 2010). D'autre part, des travaux montrent que les horaires flexibles ont pour pendant une intensification du travail (Kelliher et Anderson, 2008). Nous souhaitons explorer ces potentiels effets pervers des horaires flexibles.

Nous présenterons, dans un premier temps, notre cadre théorique, dans un second temps, notre méthodologie et nos résultats. Nous terminerons par une discussion de nos résultats.

1 Le temps de travail des femmes se comprend ici comme la somme des temps de travail professionnel et domestique. 


\section{Cadre théorique}

Notre cadre théorique s'appuie sur le modèle d'ajustement de l'individu à son travail (Pierce et Newstrom, 1980), issu de la psychologie, tout en y intégrant les apports des littératures sociologiques et gestionnaires sur le sujet. En effet, le modèle de Pierce et Newstrom (1980) envisage deux types d'ajustement individuel: le premier a trait à l'ajustement entre les aptitudes du salarié et celles exigées par l'environnement de travail ; le second porte sur l'ajustement opéré entre le système d'incitation (ou de récompense) de l'organisation et les besoins du salarié. Alors que le premier mécanisme de congruence diminue la non-satisfaction au travail pour le salarié, le second mécanisme conduit le salarié à être satisfait dans son travail. Selon Pierce et Newstrom (1980), les horaires flexibles peuvent jouer à la fois sur le premier type de convergence en réduisant le niveau de stress et les troubles du rythme circadien $^{2}$ et sur le second type de convergence, en répondant au besoin d'autonomie du salarié dans la gestion de son temps de travail.

\subsection{L'effet des horaires flexibles sur l'articulation travail-famille}

Peu de recherches ont été effectuées sur le sujet. Certaines portent sur les pratiques facilitant l'articulation travail-famille. D'autres portent spécifiquement sur les horaires flexibles. Toutes concluent à des résultats divergents sur la relation entre le bénéfice d'horaires flexibles et l'articulation entre vie privée et vie professionnelle.

Thomas et Ganster (1995) montrent, à partir d'un échantillon de 398 personnels soignants composé à $90 \%$ de femmes, que les pratiques favorisant l'articulation travail-famille, dont l'accès à des horaires flexibles, sont corrélées négativement aux conflits travail-famille. Toutefois, une recherche menée par Batt et Monique (2003), à partir d'un échantillon mixte de 557 individus, conclut que les pratiques favorisant l'articulation travail-famille ne sont pas associées significativement aux conflits travail-famille.

De même, les études portant spécifiquement sur les horaires flexibles n'aboutissent pas à des résultats convergents. Anderson et alii (2002), à partir d'un échantillon tiré d'une enquête nationale de 2877 salariés américains, concluent à une corrélation négative entre les horaires flexibles et les conflits travail-famille, sans différence significative entre les hommes et les femmes. Breaugh et Frye (2008), à partir d'un échantillon de 96 étudiants ou anciens étudiants en MBA, analysent spécifiquement l'influence des horaires flexibles sur les conflits travail-famille et concluent à une corrélation bivariée négative. Cependant, plusieurs études aboutissent à des résultats opposés. Ainsi, Lapierre et Allen (2008), à partir d'un échantillon de 230 anciens étudiants en MBA, montrent, dans le cadre d'une analyse multivariée, que les horaires flexibles ne sont pas associés significativement aux conflits travail-famille. Premeaux et alii (2008) arrivent à la même conclusion, dans le cadre d'une analyse multivariée, à partir d'un échantillon de 546 salariés répartis dans sept organisations différentes. Casper et

\footnotetext{
${ }^{2}$ C'est-à-dire le rythme biologique d'une durée de 24 heures environ.
} 
Buffardi (2004), à partir d'une expérimentation sur 371 individus consistant à leur présenter 8 vignettes décrivant différentes organisations et manipulant la flexibilité des horaires, le niveau d'aide à la garde de personnes à charge, ainsi que le niveau de salaire, concluent à une relation non significative entre la flexibilité des horaires et les conflits travail-famille. Enfin, une seule étude (Hofäcker et König, 2013), à notre connaissance, conclut à une association entre les horaires flexibles et l'articulation travail-famille différente selon le sexe des salariés. A partir d'un échantillon de 3383 salariés issus de l'enquête européenne European Social Survey (ESS), Hofäcker et König (2013) tirent le constat suivant : les femmes ont tendance à utiliser leur contrôle des heures de début et de fin d'une journée de travail pour parvenir à un meilleur équilibre entre travail et vie privée, les hommes prennent des dispositions pour accroître leur implication au travail, renforçant ainsi le conflit perçu entre travail et famille. Hofäcker et König (2013) soutiennent que cette utilisation du travail flexible selon le sexe reflète le poids des rôles genrés traditionnels et les structures sexuées du marché du travail. Nous appuyant sur la théorie de l'ajustement de Pierce et Newstrom (1980), nous pouvons dès lors supposer que les horaires flexibles vont faciliter l'ajustement entre le système d'incitation (ou de récompense) de l'organisation et les besoins du salarié en leur donnant une meilleure maitrise de leur temps et ainsi permettre une meilleure conciliation entre vie privée et vie professionnelle. Nous pouvons aussi faire l'hypothèse, sur la base des travaux de Hofäcker et König (2013), que les horaires flexibles auront un effet différencié sur la conciliation travailfamille selon le sexe. Dès lors, nous faisons l'hypothèse suivante :

Hypothèse H1: Le fait de bénéficier d'horaires flexibles améliore la conciliation travail-famille, cette amélioration travail-famille étant plus forte pour les femmes que pour les hommes.

D'un point de vue méthodologique, les études mentionnées comportent un certain nombre de limites pouvant expliquer ces résultats divergents. Tout d'abord, elles englobent dans un même concept un ensemble de pratiques gestionnaires favorisant l'articulation travail-famille. Ainsi, l'étude de Thomas et Ganster (1995) inclut dans la variable «pratiques favorisant l'articulation travail-famille » les pratiques suivantes : services de garde, information sur les services de référence (garde d'enfants, soins aux enfants malades...) et accès à des horaires flexibles. L'étude de Batt et Monique (2003) intègre dans la variable «pratiques favorisant l'articulation travail-famille » les pratiques suivantes : congé pour assurer une aide familiale, temps de garde des personnes à charge, accès à des horaires flexibles, au télétravail et à des congés pour faire du bénévolat. Cette hétérogénéité des pratiques favorisant l'articulation travail-famille limite les comparaisons possibles entre les études. Ensuite, la composition des échantillons varie fortement d'une étude à l'autre. Certains sont composés essentiellement de femmes (Thomas et Ganster, 1995 ; Breaugh et Frye, 2008) alors que d'autres sont mixtes (Batt et Monique, 2008). En outre, l'ensemble de ces études est effectué en coupe instantanée, ce qui ne permet pas d'établir de manière irréfutable une relation de causalité. Par ailleurs, ces études sont effectuées sur des échantillons de petite taille et limités à des populations spécifiques (étudiants, personnel soignant...), atténuant les validités interne et externe de leurs résultats. Enfin, ces études sont a-genrées, c'est-à-dire qu'elles ne distinguent pas les effets différenciés des horaires flexibles selon le sexe du salarié. Notre étude vise à dépasser ces différentes limites. 
Plusieurs études ont identifié un certain nombre d'effets pervers liés à l'utilisation des horaires flexibles. Tout d'abord, le fait de bénéficier d'horaires flexibles aurait pour contrepartie une intensification du travail (Kelliher et Anderson, 2008), due à une augmentation de la porosité entre les sphères privée et professionnelle (Lott, 2018; Schieman et alii, 2009). Ainsi, le fait de bénéficier d'horaires flexibles se traduirait par une augmentation des heures supplémentaires (Lott et Chung, 2016), ainsi qu'une probabilité plus forte de continuer à travailler à la maison (Lott, 2018 ; Schieman et alii, 2009). Toutefois, il existe un effet genré modérant la relation entre le fait de bénéficier d'horaires flexibles et l'intensification du travail : les femmes vont utiliser les bénéfices tirés des horaires flexibles pour améliorer l'articulation travail-famille, tandis que les hommes vont augmenter leur effort de travail lorsque les limites entre temps au travail et hors-travail disparaissent (Moen et $\mathrm{Yu}, 2000$ ). Nous faisons donc l'hypothèse suivante :

Hypothèse 2 : le fait de bénéficier d'horaires flexibles va intensifier le travail et augmenter la probabilité d'effectuer des heures supplémentaires et de continuer à travailler à la maison, cette probabilité étant plus forte pour les hommes que pour les femmes.

Un second effet pervers a été identifié dans la littérature : les horaires flexibles se traduisent par une augmentation des responsabilités familiales et domestiques prises en charge par les femmes. Les femmes utilisent les horaires flexibles pour assumer davantage de responsabilités familiales et non pour améliorer leur propre capacité à gérer le travail domestique et les exigences liées aux responsabilités familiales qu'elles assument, comme le montrent Hammer et alii (2005). Ainsi, pour les femmes, les pratiques en milieu de travail favorables à la conciliation travail-famille, telles que les horaires flexibles, peuvent accroître les attentes de leurs conjoints, ce qui accroîtra d'autant le temps qu'elles allouent aux tâches domestiques (Hammer et alii, 2005). Nous faisons ainsi l'hypothèse suivante :

Hypothèse 3 : le fait de bénéficier d'horaires flexibles va augmenter le temps alloué aux tâches domestiques, cette augmentation étant plus forte pour les femmes salariées que pour les hommes salariés.

Un troisième effet pervers lié à la mise en place des horaires flexibles est mentionné dans la littérature : il s'accompagne souvent de l'implantation de mesures visant à stimuler la performance des salariés (Felstead et Jewson, 2000) et à exercer un contrôle indirect sur leur niveau d'implication au travail. Ainsi Alber (2019, p. 22) souligne les injonctions paradoxales du néo-management : « un travailleur du privé soumis aux injonctions paradoxales du néomanagement : plus autonome, encouragé à prendre des initiatives, associé aux projets de sa structure, mais dans le même temps évalué de manière quantitative à échéances régulières et motivé par des avantages financiers ». A l'instar d'Alber (2019), nous mesurerons le contrôle indirect exercé sur les salariés via des pratiques de gestion du travail telles que des évaluations régulières, la fixation d'objectifs chiffrés à atteindre et leur niveau de difficulté, un système de 
rémunération à la performance. Ce constat sera d'autant plus vrai pour les femmes. En effet, sur la base de « suppositions sexuées » (Acker, 2006, p. 444), le fait de bénéficier d'horaires flexibles est associé pour les femmes à un «supposé » moindre engagement dans leur travail (Kronberger et alii, 2010 ; Rogier et Padgett, 2004). Nous faisons donc l'hypothèse suivante :

Hypothèse 4: le fait de bénéficier d'horaires flexibles augmente la probabilité d'être évalué et de se voir fixer des objectifs difficiles à atteindre ainsi que de percevoir une rémunération liée à la performance individuelle, cette augmentation étant plus forte pour les femmes salariées que pour les hommes salariés.

Un quatrième effet pervers, recensé dans la littérature, est lié aux conséquences genrées des horaires flexibles sur la rémunération des hommes et des femmes qui en bénéficient. En effet, l'organisation genrée du travail induit une discrimination salariale systématique en défaveur des femmes (Acker, 1990). Ainsi, quand bien même, les femmes utilisent les horaires flexibles, tout comme les hommes, pour améliorer leur productivité au travail et l'amplitude horaire de leur journée, seul est reconnu financièrement l'effort supplémentaire des hommes (Lott et Chung, 2016), ce qui accroît chez les femmes la perception d'être mal payées compte tenu de l'effort fourni.

Hypothèse 5 : le fait de bénéficier d'horaires flexibles va augmenter chez les femmes salariées la perception d'être mal rémunérées ainsi que la perception de ne pas recevoir le respect et l'estime que mérite son travail, et ce, contrairement aux hommes.

Enfin, les horaires flexibles peuvent se transformer en un «stigmate», au sens de Goffman (1968), différenciant les salariés engagés dans leur travail de ceux qui ne le sont pas (Kornberger et alii, 2010; Williams et alii, 2013). En effet, les managers perçoivent les femmes bénéficiant d'horaires flexibles comme étant moins engagées dans leur travail et dans leur carrière et comme étant moins motivées que les femmes ayant un horaire fixe (Rogier et Padgett, 2004). A l'inverse, pour les salariés hommes en bénéficiant, les horaires flexibles sont considérés par les managers comme un moyen de promouvoir leur carrière (Brescoll et alii, 2013). En outre, les salariés auxquels sont proposés une mesure facilitant l'articulation travail-famille comme le congé parental déclarent qu'ils peuvent mettre leur carrière en péril à long terme en l'utilisant (Thompson et alii, 1999). Ainsi, nous pouvons faire l'hypothèse que :

Hypothèse 6: le fait de bénéficier d'horaires flexibles va augmenter la perception que leurs perspectives de promotion sont insatisfaisantes, chez les femmes salariées.

\section{Méthodologie}

\subsection{Présentation des enquêtes Conditions de Travail 2013 et 2016}


Afin de tester nos hypothèses, nous avons utilisé les enquêtes nationales Conditions de Travail (CT) 2013 et Conditions de travail-Risques Psychosociaux (CT-RPS) 2016 appariées en un panel. Cette enquête est réalisée en « face à face » auprès d'un échantillon d'environ 28 000 enquêtés. C'est la seule enquête française sur les conditions de travail de cette ampleur et portant sur l'ensemble des actifs occupés. L'échantillon en panel est composé de 19882 individus actifs occupés qui ont répondu à la première vague de l'enquête CT 2013 puis ont participé à la deuxième vague de l'enquête CT-RPS 2016. Les enquêtes CT 2013 et CT-RPS 2016 ont été menées par la direction de l'Animation de la recherche, des Études et des Statistiques (Dares), en collaboration avec la direction générale de l'Administration et de la Fonction publique (DGAFP), la direction de la Recherche, des Études, de l'Evaluation et des Statistiques (Drees) du ministère de la Santé et l'INSEE. L'enquête Conditions de travailRisques Psychosociaux (CT-RPS) 2016 permet de mesurer l'évolution des conditions de travail - les deux tiers des questions sont identiques à celles de l'enquête CT 2013 - et d'approfondir l'analyse des risques psychosociaux

L'utilisation de ce panel nous donne la possibilité d'effectuer des analyses longitudinales. Ces analyses nous permettent d'établir statistiquement des relations causales entre nos variables exogènes et endogènes. Les travailleurs indépendants $(n=1689)$ ont été exclus d'emblée de notre analyse. Compte tenu de l'intérêt porté à l'articulation travail-famille, seuls les salariés âgés de 30 à 50 ans, soit ceux qui sont les plus susceptibles d'avoir des enfants à charge (l'âge moyen pour un premier enfant étant de 28,5 ans en 2015 en France $^{3}$ ), ont été retenus. Par ailleurs, les individus ayant changé d'établissement entre l'enquête CT 2013 et l'enquête CTRPS 2016 ( $n=2792)$ ont été exclus de notre échantillon. Notre échantillon final contient donc 9555 individus actifs occupés, dont 4071 hommes et 5484 femmes.

2.2 Mesures de la variable indépendante et des variables dépendantes

\section{La variable exogène}

La flexibilité des horaires est une variable nominale mesurée au travers des réponses à la question suivante : «Comment sont déterminés vos horaires ?». Les participants pouvaient choisir entre quatre catégories : (1) «Ils sont déterminés par l'entreprise ou l'administration sans possibilité de modification» (horaires fixes); (2) «Vous pouvez choisir entre plusieurs horaires fixes proposés par l'entreprise ou l'administration» (choix des horaires fixes); (3) «Vos horaires sont modifiables par vous-même d'un jour à l'autre, dans un système du type horaires à la carte» (horaires flexibles ou « horaires à la carte»); (4) «Vos horaires sont déterminés par vous-même » (horaires libres). Cette variable a été traitée comme une variable catégorielle et codée 4 pour la modalité « horaires fixes », 3 pour « choix des horaires fixes », 2 pour « horaires flexibles », 1 pour « horaires libres ». Cette variable n'est présente que dans l'enquête Conditions de travail 2013.

\section{Les variables endogènes}


La conciliation vie privée-vie professionnelle (CVFVP) est une variable ordinale mesurée au travers des réponses à la question suivante: «En général, vos horaires de travail s'accordent-ils avec vos engagements sociaux et familiaux en dehors de votre travail ? ». Les réponses s'étalent sur un continuum à 4 points allant de «Pas du tout » (codée 1) à «Très bien » (codée 4).

Le travail domestique (DOMES) est une variable ordinale mesurée au travers des réponses à la question suivante: "Approximativement, combien d'heures par semaine êtes-vous impliqué(e) dans les tâches domestiques (préparation des repas, courses d'alimentation, lessive...)?». Les participants pouvaient choisir entre plusieurs catégories ordinales: « Moins de 2 heures » (codée 1), « entre 3 et 6 heures » (codée 2), « entre 7 et 9 heures (codée 3 ), « entre 10 et 12 heures » (codée 4), « plus de 12 heures » (codée 5). Cette variable n'est présente que dans l'enquête Conditions de travail-RPS 2016.

La variable "perspectives de promotion " (PROMOTION) est une variable ordinale mesurée au travers des réponses à la question suivante: « $\mathrm{Vu}$ tous mes efforts, mes perspectives de promotion sont satisfaisantes ». Les réponses s'étalent sur un continuum à 4 points allant de « Pas du tout d'accord » (codée 1) à « Tout à fait d'accord » (codée 4).

La variable « estime perçue » (MERITE) est une variable ordinale mesurée au travers des réponses à la question suivante : «Vu tous mes efforts, je reçois le respect et l'estime que mérite mon travail ». Les réponses s'étalent sur un continuum à 4 points allant de «Pas du tout d'accord » (codée 1) à « Tout à fait d'accord» (codée 4).

Les heures supplémentaires effectuées (HSUP) est une variable ordinale mesurée au travers des réponses à la question suivante : « Vous arrive-t-il de travailler au-delà de l'horaire prévu ?». Les réponses s'étalent sur un continuum à 4 points allant de «Jamais » (codée 1) à « Tous les jours » (codée 4).

Le fait de continuer à travailler à la maison (MAISON) est une variable ordinale mesurée au travers des réponses à la question suivante : «Vous arrive-t-il d'emporter du travail chez vous? ». Les réponses s'étalent sur un continuum à 4 points allant de « Jamais » (codée 1) à « Tous les jours ou presque » (codée 4).

L'intensification du travail est une variable composite calculée à partir de la moyenne de la somme des scores de 4 items, dont les réponses s'étalent sur un continuum à 4 points, mesurant l'intensité du travail. Un exemple d'item est le suivant : «Etes-vous obligé de vous dépêcher dans votre travail ? ». Les cohérences internes (alpha de Cronbach) pour les mesures de l'intensification sont respectivement de 0,699 pour 2013 et de 0,716 pour 2016. Ces deux mesures de l'intensification au travail en 2013 et en 2016 ont été ensuite divisées en quartile afin de les harmoniser avec les autres mesures des variables endogènes.

Le fait d'avoir à atteindre des objectifs fixés (OBJECTIF) est une variable dichotomique mesurée au travers des réponses à la question suivante : «Devez-vous atteindre des objectifs chiffrés et précis? ». Les réponses étaient « oui » (codée 1$)$ à « non» (codée 0$)$.

Le fait d'avoir des objectifs inatteignables (OBJATTEIN) est une variable ordinale mesurée au travers des réponses à la question suivante : «Avez-vous du mal à atteindre les 
objectifs fixés ?». Les réponses s'étalent sur un continuum à 4 points allant de « Toujours » (codée 4$)$ à « jamais » (codée 1).

Le fait d'avoir au moins un entretien d'évaluation par an (EVA) est une variable dichotomique mesurée au travers des réponses à la question suivante : « Avez-vous au moins un entretien d'évaluation par an? ». Les réponses étaient « oui » (codée 1) à « non» (codée 0).

Le fait de voir sa rémunération liée à ses performances individuelles (REMPERF) est une variable ordinale mesurée au travers des réponses à la question suivante : «Dans quelle mesure votre rémunération annuelle dépend-elle de vos performances ?». Les réponses s'étalent sur un continuum à 3 points allant de «Pas du tout » (codée 1) à «Dans une forte mesure » (codée 3$)$.

Le fait de trouver son travail bien payé (PAYECOM) est une variable ordinale mesurée au travers des réponses à la question suivante: "Compte tenu du travail que vous réalisez, diriez-vous que vous êtes?». Les réponses s'étalent sur un continuum à 5 points allant de « Très mal payé » (codée 5) à « Très bien payé » (codée 1$)$.

\section{Les variables de contrôle}

Nous avons introduit des variables de contrôle afin d'éviter les effets de confusion. Ces derniers consistent à attribuer la cause d'un effet à la variable explicative testée (dans notre cas, la flexibilité des horaires) alors que cet effet est expliqué par une autre ou d'autres variables (Mbengue et Vandangeon-Derumez, 2003). Nous avons donc contrôlé les effets d'un ensemble de variables qui peuvent être des déterminants de l'implantation d'horaires flexibles tout en ayant des effets sur nos variables dépendantes. Nous avons introduit dans notre modèle les variables de contrôle suivantes : âge, catégorie socio-professionnelle, type de temps de travail (temps partiel ou complet), type de contrat de travail (CDI, CDD/intérim, stagiaire), statut marital (célibataire ou en couple), nombre d'enfants âgés de moins de 3 ans, nombre d'enfants âgés entre 3 et 17 ans, taille de l'établissement (nombre de salariés), secteur d'activité (nomenclature de l'INSEE distinguant 17 secteurs d'activité).

\subsection{Analyses statistiques}

L'échantillon sur lequel nous appuyons nos analyses statistiques est en panel et constitué de deux vagues : les années 2013 et 2016. Pour éviter tout biais d'endogénéité, lié à l'omission d'une variable à fort pouvoir explicatif (Greene, 2003), nous avons inclus une variable endogène décalée $Y_{t-1}$, à l'instar de Kelly et alii (2011). Compte tenu de la nature ordinale ou dichotomique des variables endogènes, nous avons opté pour des modèles de régressions logistiques ordinale ou dichotomique incluant une variable endogène décalée $Y_{t-1}$ ainsi que des variables exogènes décalées $X_{t-1}$, incluant la variable explicative principale, la flexibilité des horaires de travail, et des variables de contrôle. Le modèle statistique prend donc la forme suivante : 


$$
Y_{t}=g\left(a . Y_{t-1}+b^{T} \cdot X_{t-1}\right)+\varepsilon_{t-1}
$$

avec $Y_{t}$ la variable endogène en $\mathrm{t}$, soit 2016, $Y_{t-1}$, la variable endogène décalée en $\mathrm{t}-1$, soit 2013, $X_{t-1}$, le vecteur des variables exogènes décalées à la période $\mathrm{t}-1$, soit $2013, b^{T}$, le vecteur transposé des pondérations des variables exogènes, $\varepsilon_{t-1}$, le terme d'erreur à la période $\mathrm{t}-1$, soit 2013 et $g$, la fonction logistique ordinale ou dichotomique.

Afin de tenir compte des analyses de Kergoat (1978) proposant une articulation théorique de deux variables, le sexe et les classes sociales, nous avons effectué nos analyses statistiques sur des sous-échantillons composés, soit d'hommes ou de femmes non-cadres, soit d'hommes ou de femmes cadres.

\section{Résultats}

Nous présentons dans le tableau 1 la ventilation des formes de flexibilité du temps de travail en fonction du sexe et de la catégorie socio-professionnelle (cadre/non-cadre). Ces résultats montrent que la flexibilité du temps de travail est sexuée et fonction de l'appartenance à une classe sociale, rejoignant en cela les analyses de Kergoat (1978) sur la juxtaposition d'une division technique et sociale du travail à une division sexuelle du travail. Ainsi, si l'on se focalise sur la seule classe sociale des cadres, si 37,5\% des hommes cadres déclarent bénéficier d'horaires libres, 17,2\% d'horaires à la carte, seules respectivement 18,8 \% et 13,9 $\%$ des femmes cadres déclarent être dans ce cas. A l'inverse, si l'on se focalise sur la classe sociale des non-cadres (c'est-à-dire les ouvriers et les employés), les femmes non-cadres déclarent plus que les hommes non-cadres bénéficier des différentes formes de flexibilité du temps de travail (horaires libres, horaires à la carte, choix entre plusieurs horaires fixes). Logiquement, les hommes non-cadres déclarent majoritairement être soumis à des horaires fixes standards $(74,8 \%$ contre $66 \%$ des femmes non-cadres $)$.

Tableau 1. Ventilation en \% des formes de flexibilité du temps de travail en fonction du sexe et de la catégorie socio-professionnelle (cadre/non-cadre)

\begin{tabular}{lcccc}
\hline & \multicolumn{2}{c}{ Hommes } & \multicolumn{2}{c}{ Femmes } \\
\cline { 2 - 5 } & Non-cadres & Cadres & Non-cadres & Cadres \\
Horaires libres & 10,2 & 37,5 & 11,1 & 18,8 \\
$\begin{array}{l}\text { Horaires à la } \\
\text { carte }\end{array}$ & 7,7 & 17,2 & 10,3 & 13,9
\end{tabular}


Choix entre 7,3

12,5

14,4

plusieurs horaires

fixes

Horaires fixes

74,8

31,9

66,0

52,8

$\mathrm{N}$

3086

985

4281

1203

Source : Enquête Conditions de Travail 2013.

Note : les pourcentages sont pondérés en fonction d'un coefficient calculé afin que les répondants soient représentatifs de l'ensemble des actifs en emploi en France métropolitaine et dans les quatre départements d'Outre-mer couverts

Nous avons tout d'abord testé les effets de la flexibilité des horaires de travail sur la conciliation entre vie familiale et vie professionnelle (CVFVP). Nos résultats sont présentés dans le tableau 2. L'hypothèse 1 est infirmée. En effet, deux formes de flexibilité des horaires (« horaires à la carte » et « choix entre plusieurs horaires ») améliorent l'articulation travail-famille, de manière plus importante ${ }^{4}$, pour les hommes non-cadres que pour les femmes non-cadres. Les horaires libres contribuent à améliorer l'articulation travail-famille pour les seules femmes non-cadres. Contrairement à l'effet attendu, la flexibilité des horaires et plus précisément, la modalité « choix entre plusieurs horaires fixes »- dégrade l'articulation travail-famille pour les femmes cadres.

Tableau 2. Les effets de la présence de la flexibilité des horaires en 2013 sur la CVFVP en 2016 : résultats de régressions logistiques ordinales (Paramètres estimés et test de Wald)

\begin{tabular}{|c|c|c|c|c|}
\hline & \multicolumn{2}{|c|}{ Hommes } & \multicolumn{2}{|c|}{ Femmes } \\
\hline & Cadres & Non-cadres & Cadres & Non-cadres \\
\hline$C V F V P_{2013}$ & $1,181 * * *$ & $1,143 * * *$ & $1,208 * * *$ & $1,229 * * *$ \\
\hline Horaires libres & $-0,182(\mathrm{~ns})$ & 0,157 (ns) & $-0,318(\mathrm{~ns})$ & $0,344^{*}$ \\
\hline $\begin{array}{l}\text { Horaires à la } \\
\text { carte }\end{array}$ & $0,134(\mathrm{~ns})$ & $0,415 * *$ & 0,198 (ns) & $0,382 * * *$ \\
\hline $\begin{array}{l}\text { Choix entre } \\
\text { plusieurs horaires } \\
\text { fixes }\end{array}$ & 0,101 (ns) & $0,428 * *$ & $-0,459 * *$ & $0,363 * * *$ \\
\hline $\mathrm{R}^{2}$ Cox et Snell & 0,224 & 0,216 & 0,224 & 0,230 \\
\hline
\end{tabular}

4 Le poids des coefficients associés aux modalités « horaires à la carte » et « choix entre plusieurs horaires » est plus élevé pour les hommes que pour les femmes. 
Nous avons ensuite testé les effets de la flexibilité des horaires sur la probabilité d'effectuer des heures supplémentaires (tableau 3a) ainsi que de travailler à la maison (tableau 3b). L'hypothèse 2 est partiellement vérifiée. Nous observons un effet genré très marqué concernant la relation entre la flexibilité des horaires et la probabilité d'effectuer des heures supplémentaires. Ainsi, si les horaires à la carte augmentent la probabilité d'effectuer des heures supplémentaires pour les non-cadres masculins (tableau 3a), ils ont l'effet inverse sur les non-cadres féminins. De même, les horaires libres augmentent la probabilité d'effectuer des heures supplémentaires pour les non-cadres hommes, ce qui n'est pas le cas des noncadres femmes (tableau 3a). Nous observons le même effet genré de la flexibilité des horaires sur la probabilité d'emporter du travail à la maison. Les horaires libres vont diminuer la probabilité d'emporter du travail à la maison pour les cadres femmes, alors que la flexibilité des horaires, quel qu'en soit sa forme, n'a pas de relation significative avec la probabilité d'emporter du travail à la maison pour les hommes cadres et non-cadres (tableau 3b). Toutefois, contrairement à l'effet attendu, les horaires à la carte contribuent à augmenter l'intensité du travail pour les femmes cadres tandis que les horaires libres la diminuent pour les femmes non-cadres. Aucune relation significative n'est observée entre la flexibilité des horaires et l'intensification du travail pour les hommes cadres et non-cadres (tableau $3 \mathrm{c}$ ).

Tableau 3a. Les effets de la présence de la flexibilité des horaires en 2013 sur la probabilité d'effectuer des heures (HSUP) en 2016 : résultats de régressions logistiques ordinales (Paramètres estimés et test de Wald)

\begin{tabular}{|c|c|c|c|c|}
\hline & & Imes & & mes \\
\hline & Cadres & Non-cadres & Cadres & Non-cadres \\
\hline$H S U P_{2013}$ & $0,720 * * *$ & $1,286 * * *$ & $0,953 * * *$ & $1,327 * * *$ \\
\hline Horaires libres & 0,277 (ns) & $0,483^{* *}$ & $0,106(\mathrm{~ns})$ & $-0,016(\mathrm{~ns})$ \\
\hline $\begin{array}{l}\text { Horaires à la } \\
\text { carte }\end{array}$ & $-0,075(\mathrm{~ns})$ & $0,439^{* *}$ & 0,034 (ns) & $-0,262 *$ \\
\hline $\begin{array}{l}\text { Choix entre } \\
\text { plusieurs horaires } \\
\text { fixes }\end{array}$ & $0,158(\mathrm{~ns})$ & $0,031(\mathrm{~ns})$ & $-0,099$ (ns) & $0,082(\mathrm{~ns})$ \\
\hline $\mathrm{R}^{2}$ Cox et Snell & 0,136 & 0,249 & 0,190 & 0,249 \\
\hline
\end{tabular}

Tableau 3b. Les effets de la présence de la flexibilité des horaires en 2013 sur la probabilité d'emporter du travail à la maison (MAISON) en 2016 : résultats de régressions logistiques ordinales (Paramètres estimés et test de Wald)

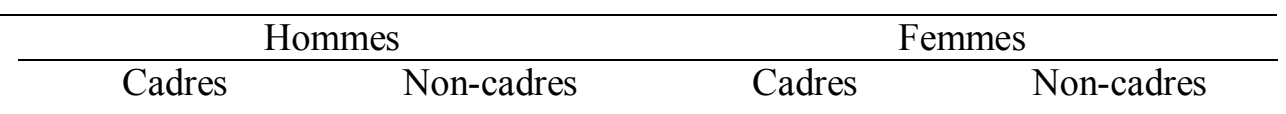




\begin{tabular}{|c|c|c|c|c|}
\hline$M_{A I S O N} 2013$ & $1,859 * * *$ & $1,784 * * *$ & $2,366^{* * *}$ & $2,030 * * *$ \\
\hline Horaires libres & 0,249 (ns) & $-0,036$ (ns) & $-0,452^{*}$ & 0,273 (ns) \\
\hline $\begin{array}{l}\text { Horaires à la } \\
\text { carte }\end{array}$ & - 0,038 (ns) & $-0,162$ (ns) & $0,051(\mathrm{~ns})$ & 0,195 (ns) \\
\hline $\begin{array}{l}\text { Choix entre } \\
\text { plusieurs horaires } \\
\text { fixes }\end{array}$ & $-0,362$ (ns) & $0,250(\mathrm{~ns})$ & $-0,021(\mathrm{~ns})$ & 0,008 (ns) \\
\hline $\mathrm{R}^{2}$ Cox et Snell & 0,568 & 0,341 & 0,677 & 0,373 \\
\hline
\end{tabular}

Tableau 3c. Les effets de la présence de la flexibilité des horaires en 2013 sur l'intensification du travail (INTENSITE) en 2016 : résultats de régressions logistiques ordinales (Paramètres estimés et test de Wald)

\begin{tabular}{|c|c|c|c|c|}
\hline & \multicolumn{2}{|c|}{ Hommes } & \multicolumn{2}{|c|}{ Femmes } \\
\hline & Cadres & Non-cadres & Cadres & Non-cadres \\
\hline INTENSITE $E_{2013}$ & $0,965 * * *$ & $0,889 * * *$ & $0,959 * * *$ & $0,916^{* * *}$ \\
\hline Horaires libres & $0,230(\mathrm{~ns})$ & $-0,125(\mathrm{~ns})$ & $0,082(\mathrm{~ns})$ & $-0,240(\mathrm{~ns})$ \\
\hline Horaires à la carte & 0,165 (ns) & $-0,212(\mathrm{~ns})$ & $0,521 * *$ & $0,002(\mathrm{~ns})$ \\
\hline $\begin{array}{l}\text { Choix entre } \\
\text { plusieurs horaires } \\
\text { fixes }\end{array}$ & $-0,112(\mathrm{~ns})$ & $-0,150(\mathrm{~ns})$ & $-0,059(\mathrm{~ns})$ & $-0,097(\mathrm{~ns})$ \\
\hline $\mathrm{R}^{2}$ Cox et Snell & 0,315 & 0,291 & 0,115 & 0,118 \\
\hline
\end{tabular}

Nous avons ensuite testé l'effet de la flexibilité des horaires sur le temps de travail domestique (tableau 4). Rappelons que la variable DOMEST n'était pas mesurée dans l'enquête Conditions de Travail 2013, nous n'avons donc pas pu mesurer l'effet retardé $D_{O M E S T} 2013$ pour cette variable. L'hypothèse 3 est infirmée. Contrairement à l'effet attendu, le fait de bénéficier d'horaires à la carte diminue le temps de travail domestique pour les non-cadres femmes. 
Tableau 4. Les effets de la présence de la flexibilité des horaires en 2013 sur le temps de travail domestique (DOMEST) en 2016 : résultats de régressions logistiques ordinales (Paramètres estimés et test de Wald)

\begin{tabular}{|c|c|c|c|c|}
\hline & \multicolumn{2}{|c|}{ Hommes } & \multicolumn{2}{|c|}{ Femmes } \\
\hline & Cadres & Non-cadres & Cadres & $\begin{array}{l}\text { Non- } \\
\text { cadres }\end{array}$ \\
\hline Horaires libres & $-0,307^{*}$ & $-0,231$ (ns) & $-0,275$ (ns) & $-0,039$ (ns) \\
\hline Horaires à la carte & $-0,196(\mathrm{~ns})$ & 0,009 (ns) & $-0,249$ (ns) & $-0,249 *$ \\
\hline $\begin{array}{l}\text { Choix entre } \\
\text { plusieurs horaires } \\
\text { fixes }\end{array}$ & 0,045 (ns) & 0,101 (ns) & 0,038 (ns) & 0,036 (ns) \\
\hline $\mathrm{R}^{2}$ Cox et Snell & 0,081 & 0,048 & 0,112 & 0,073 \\
\hline
\end{tabular}

Nous avons ensuite testé si le fait de bénéficier d'horaires flexibles augmente la probabilité d'être évalué et d'avoir des objectifs fixés à atteindre ainsi que de voir sa rémunération dépendre de son niveau individuel de performance (tableaux 5a, 5b et 5c). Rappelons que la variable REMPERF n'était pas mesurée dans l'enquête Conditions de Travail 2013, nous n'avons donc pas pu l'effet retardé $R E M P E R F_{2013}$ pour cette variable. L'hypothèse 4 est vérifiée pour les cadres. Les horaires libres augmentent la probabilité d'avoir des objectifs chiffrés précis à atteindre pour les femmes, de manière plus importante que pour les hommes (tableau 5a). Toutefois, la flexibilité des horaires n'a pas d'effet sur la difficulté des objectifs à atteindre, ce quel que soit le sexe du salarié (tableau 5b). L'effet des horaires flexibles sur la probabilité d'être évalué est intersectionnel (c'est-à-dire qu'il se situe à l'intersection des effets du sexe et de la catégorie socio-professionnelle). Ainsi, les horaires flexibles (et plus précisément les horaires à la carte) accroissent la probabilité d'être évalué au moins une fois par an pour les femmes cadres, à l'exclusion des femmes non-cadres. A l'inverse, les horaires flexibles (et plus précisément, le choix entre plusieurs horaires) accroissent la probabilité d'être évalué au moins une fois par an pour les hommes non-cadres, à l'exclusion des hommes cadres (tableau 5c). La flexibilité des horaires, quel qu'en soit la forme, augmente la probabilité de voir sa rémunération dépendre de sa performance individuelle pour les cadres hommes et femmes. Les horaires libres augmentent de même cette probabilité pour les non-cadres hommes. La flexibilité des horaires n'a aucune influence significative sur la probabilité de voir sa rémunération dépendre de sa performance individuelle pour les non-cadres femmes (tableau $5 \mathrm{~d}$ ). 
Tableau 5a. Les effets de la présence de la flexibilité des horaires en 2013 sur la probabilité d'avoir des objectifs chiffrés précis à atteindre (OBJECTIF) en 2016 : résultats de régressions logistiques dichotomiques (Paramètres estimés et test de Wald)

\begin{tabular}{|c|c|c|c|c|}
\hline & \multicolumn{2}{|c|}{ Hommes } & \multicolumn{2}{|c|}{ Femmes } \\
\hline & Cadres & Non-cadres & Cadres & Non-cadres \\
\hline$O B J E C T I F_{2013}$ & $2,381 * * *$ & $2,007 * * *$ & $2,119 * * *$ & $2,219 * * *$ \\
\hline Horaires libres & $0,575^{* *}$ & $-0,029(\mathrm{~ns})$ & $0,590 * *$ & $-0,380(\mathrm{~ns})$ \\
\hline $\begin{array}{l}\text { Horaires à la } \\
\text { carte }\end{array}$ & $0,020(\mathrm{~ns})$ & $0,102(\mathrm{~ns})$ & $0,484 *$ & $0,294 *$ \\
\hline $\begin{array}{l}\text { Choix entre } \\
\text { plusieurs horaires } \\
\text { fixes }\end{array}$ & $-0,037(\mathrm{~ns})$ & $-0,167(\mathrm{~ns})$ & 0,279 (ns) & $0,297 *$ \\
\hline $\mathrm{R}^{2}$ Cox et Snell & 0,165 & 0,162 & 0,140 & 0,193 \\
\hline
\end{tabular}

Tableau 5b. Les effets de la présence de la flexibilité des horaires en 2013 sur la probabilité d'avoir des objectifs fixés difficiles à atteindre (OBJATTEINT) en 2016 : résultats de régressions logistiques ordinales (Paramètres estimés et test de Wald)

\begin{tabular}{|c|c|c|c|c|}
\hline & \multicolumn{2}{|c|}{ Hommes } & \multicolumn{2}{|c|}{ Femmes } \\
\hline & Cadres & Non-cadres & Cadres & Non-cadres \\
\hline OBJATTEINT 2013 & $0,737 * * *$ & $0,765 * * *$ & $0,452 * * *$ & $0,760 * * *$ \\
\hline Horaires libres & $-0,246(\mathrm{~ns})$ & $0,552(\mathrm{~ns})$ & 0,078 (ns) & $0,334(\mathrm{~ns})$ \\
\hline Horaires à la carte & $-0,367(\mathrm{~ns})$ & $-0,303(\mathrm{~ns})$ & 0,192 (ns) & $0,344(\mathrm{~ns})$ \\
\hline $\begin{array}{l}\text { Choix entre } \\
\text { plusieurs horaires } \\
\text { fixes }\end{array}$ & $-0,184(\mathrm{~ns})$ & $-0,331(\mathrm{~ns})$ & 0,156 (ns) & $-0,344(\mathrm{~ns})$ \\
\hline $\mathrm{R}^{2}$ Cox et Snell & 0,165 & 0,162 & 0,140 & 0,193 \\
\hline
\end{tabular}


Tableau 5c. Les effets de la présence de la flexibilité des horaires en 2013 sur la probabilité d'avoir au moins un entretien d'évaluation par an (EVA) en 2016 : résultats de régressions logistiques dichotomiques (Paramètres estimés et test de Wald)

\begin{tabular}{|c|c|c|c|c|}
\hline & \multicolumn{2}{|c|}{ Hommes } & \multicolumn{2}{|c|}{ Femmes } \\
\hline & Cadres & Non-cadres & Cadres & Non-cadres \\
\hline$E V A_{2013}$ & $3,282 * * *$ & $2,569 * * *$ & $3,894 * * *$ & $2,507 * * *$ \\
\hline Horaires libres & 0,216 (ns) & $-0,225$ (ns) & 0,377 (ns) & $-0,287(\mathrm{~ns})$ \\
\hline $\begin{array}{l}\text { Horaires à la } \\
\text { carte }\end{array}$ & $-0,088$ (ns) & 0,348 (ns) & $0,744 * *$ & 0,080 (ns) \\
\hline $\begin{array}{l}\text { Choix entre } \\
\text { plusieurs horaires } \\
\text { fixes }\end{array}$ & $0,051(\mathrm{~ns})$ & $0,444^{*}$ & 0,445 (ns) & 0,349 (ns) \\
\hline $\mathrm{R}^{2}$ Cox et Snell & 0,165 & 0,162 & 0,140 & 0,193 \\
\hline
\end{tabular}

Tableau 5d. Les effets de la présence de la flexibilité des horaires en 2013 sur la probabilité d'avoir sa rémunération dépendre de sa performance (REMPERF) en 2016 : résultats de régressions logistiques ordinales (Paramètres estimés et test de Wald)

\begin{tabular}{|c|c|c|c|c|}
\hline & \multicolumn{2}{|c|}{ Hommes } & \multicolumn{2}{|c|}{ Femmes } \\
\hline & Cadres & Non-cadres & Cadres & Non-cadres \\
\hline Horaires libres & $0,893 * * *$ & $0,671 * * *$ & $0,720 * * *$ & 0,287 (ns) \\
\hline $\begin{array}{l}\text { Horaires à la } \\
\text { carte }\end{array}$ & $0,569 * *$ & 0,241 (ns) & $0,462 *$ & 0,262 (ns) \\
\hline $\begin{array}{l}\text { Choix entre } \\
\text { plusieurs horaires } \\
\text { fixes }\end{array}$ & $0,754 * * *$ & 0,164 (ns) & $0,476^{*}$ & 0,183 (ns) \\
\hline $\mathrm{R}^{2}$ Cox et Snell & 0,222 & 0,085 & 0,126 & 0,038 \\
\hline
\end{tabular}

Tableau 6a. Les effets de la présence de la flexibilité des horaires en 2013 sur la perception d'être mal payée (PAYECOM) en 2016 : résultats de régressions logistiques ordinales (Paramètres estimés et test de Wald)

\begin{tabular}{lccc} 
& Hommes & & Femmes \\
\hline Cadres & Non-cadres & Cadres & Non-cadres
\end{tabular}




\begin{tabular}{|c|c|c|c|c|}
\hline$P_{A Y E C O M} 2013$ & $1,749 * * *$ & $1,287 * * *$ & $1,467 * * *$ & $1,465 * * *$ \\
\hline Horaires libres & 0,008 (ns) & 0,068 (ns) & $-0,273$ (ns) & $-0,458 * * *$ \\
\hline $\begin{array}{l}\text { Horaires à la } \\
\text { carte }\end{array}$ & - 0,240 (ns) & $-0,230$ (ns) & $-0,067$ (ns) & $-0,091(\mathrm{~ns})$ \\
\hline $\begin{array}{l}\text { Choix entre } \\
\text { plusieurs horaires } \\
\text { fixes }\end{array}$ & 0,050 (ns) & $-0,128$ (ns) & $-0,131$ (ns) & $-0,101(\mathrm{~ns})$ \\
\hline $\mathrm{R}^{2}$ Cox et Snell & 0,192 & 0,244 & 0,314 & 0,301 \\
\hline
\end{tabular}

Pour les femmes salariées, nous avons analysé la relation entre le bénéfice d'horaires flexibles et la perception d'être mal rémunérées ainsi que celle de ne pas recevoir le respect et l'estime mérités par leur travail (tableaux 6a et 6b). L'hypothèse 5 est infirmée. Contrairement à l'effet attendu, pour les femmes non-cadres, les horaires libres diminuent la probabilité de s'estimer mal rémunérées. La flexibilité des horaires n'a aucun effet significatif sur la probabilité de s'estimer mal rémunérés pour les hommes cadres et non-cadres (tableau 6a). De même, le fait de pouvoir choisir entre plusieurs horaires fixes augmente la perception de recevoir le respect et l'estime mérités pour les femmes non-cadres. Les horaires libres ont le même effet pour les cadres masculins.

Tableau 6b. Les effets de la présence de la flexibilité des horaires en 2013 sur la perception de recevoir le respect et l'estime (MERITE) en 2016 : résultats de régressions logistiques ordinales (Paramètres estimés et test de Wald) 


\begin{tabular}{|c|c|c|c|c|}
\hline & Cadres & Non-cadres & Cadres & Non-cadres \\
\hline$M E R I T E_{2013}$ & $1,080 * * *$ &, $825 * * *$ & $0,881 * * *$ & $0,802 * * *$ \\
\hline Horaires libres & $0,375^{*}$ & 0,241 (ns) & 0,097 (ns) & 0,145 (ns) \\
\hline $\begin{array}{l}\text { Horaires à la } \\
\text { carte }\end{array}$ & $0,350(\mathrm{~ns})$ & 0,219 (ns) & $-0,226$ (ns) & 0,060 (ns) \\
\hline $\begin{array}{l}\text { Choix entre } \\
\text { plusieurs horaires } \\
\text { fixes }\end{array}$ & 0,236 (ns) & 0,220 (ns) & 0,110 (ns) & $0,191^{*}$ \\
\hline $\mathrm{R}^{2}$ Cox et Snell & 0,208 & 0,142 & 0,142 & 0,132 \\
\hline
\end{tabular}

Pour les femmes salariées, nous avons enfin testé les effets des horaires flexibles sur la perception de perspectives de promotion insatisfaisantes, contrairement aux perspectives masculines (tableau 7). L'hypothèse 6 est confirmée. Le fait de bénéficier d'horaires à la carte diminue la perception que leurs perspectives de promotion sont satisfaisantes pour les femmes non-cadres. A l'inverse, les horaires libres augmentent la perception que leurs perspectives de promotion sont satisfaisantes pour les hommes non-cadres.

Tableau 7. Les effets de la présence de la flexibilité des horaires en 2013 sur la perception d'être promu (PROMOTION) en 2016 : résultats de régressions logistiques ordinales (Paramètres estimés et test de Wald)

\begin{tabular}{|c|c|c|c|c|}
\hline & \multicolumn{2}{|c|}{ Hommes } & \multicolumn{2}{|c|}{ Femmes } \\
\hline & Cadres & Non-cadres & Cadres & Non-cadres \\
\hline PROMOTION 2013 & $1,384 * * *$ &, $896 * * *$ & $1,212 * * *$ & $1,025 * * *$ \\
\hline Horaires libres & $0,101(\mathrm{~ns})$ & $0,466^{* *}$ & $0,135(\mathrm{~ns})$ & $0,023(\mathrm{~ns})$ \\
\hline Horaires à la carte & $-0,062(\mathrm{~ns})$ & $0,153(\mathrm{~ns})$ & $-0,302(\mathrm{~ns})$ & $-0,245^{*}$ \\
\hline $\begin{array}{l}\text { Choix entre } \\
\text { plusieurs horaires } \\
\text { fixes }\end{array}$ & $-0,277(\mathrm{~ns})$ & $0,084(\mathrm{~ns})$ & $0,048(\mathrm{~ns})$ & $-0,019(\mathrm{~ns})$ \\
\hline $\mathrm{R}^{2}$ Cox et Snell & 0,298 & 0,167 & 0,237 & 0,203 \\
\hline
\end{tabular}

Le tableau 8 récapitulatif présente les hypothèses validées ou infirmées. 


\section{Hypothèses validées ou infirmées}

H1 : Le fait de bénéficier d'horaires flexibles améliore la conciliation travail-famille, cette amélioration travail-famille est d'autant plus forte pour les femmes.

H2 : le fait de bénéficier d'horaires flexibles va intensifier le travail et augmenter la probabilité d'effectuer des heures supplémentaires et de continuer à travailler à la maison, cette probabilité étant plus forte pour les hommes que pour les femmes.

H3 : le fait de bénéficier d'horaires flexibles va augmenter le temps alloué aux tâches domestiques, cette augmentation étant plus forte pour les femmes salariées que pour les hommes salariés.

H4 : le fait de bénéficier d'horaires flexibles va augmenter la probabilité d'être évalué et d'avoir des objectifs fixés à atteindre ainsi que de voir sa rémunération dépendre de son niveau individuel de performance, et ce, indifféremment du sexe du salarié.

H5 : le fait de bénéficier d'horaires flexibles va augmenter chez les femmes salariées la perception d'être mal payée ainsi que la perception de ne pas recevoir le respect et l'estime que mérite son travail, et ce, contrairement aux hommes.

H6 : le fait de bénéficier d'horaires flexibles va augmenter chez les femmes salariées la perception que leurs perspectives de promotion sont insatisfaisantes, et ce, contrairement aux hommes.
H1 infirmée

H2 partiellement validée (relation positive entre horaires à la carte et intensification du travail pour les femmes cadres)

H3 infirmée

H4 validée pour les cadres

H5 infirmée

H6 validée

\section{Discussion}

Nos résultats montrent, contrairement à des études précédentes, en coupe instantanée et ne distinguant pas à la fois le sexe et la classe sociale des salariés, que lorsque cette analyse est longitudinale et intersectionnelle, la flexibilité des horaires améliore la conciliation travailfamille des femmes non-cadres mais moins que celle des hommes non-cadres. Pis, la flexibilité des horaires dégrade la conciliation travail-famille des femmes cadres.

Plusieurs explications sont envisagées pour décrypter ces résultats inattendus. La première est celle d'un effet à double-tranchant des horaires flexibles confirmé par nos analyses. En effet, les femmes cadres bénéficiant d'horaires à la carte voient leur travail s'intensifier, contrairement aux hommes cadres. Cette intensification du travail s'explique en partie par la contrepartie importante imposée aux femmes cadres bénéficiant d'horaires à la carte sans qu'il en soit ainsi pour les hommes cadres : la mise en place d'une gestion du travail axée sur un contrôle indirect de la performance via l'imposition d'une évaluation régulière et d'une direction par objectifs. Une autre explication réside dans l'idée que les horaires flexibles proposés aux femmes ont accru les attentes que pouvaient avoir leurs conjoints vis-à-vis de leurs responsabilités familiales et domestiques, ce qui a conduit à accroître le conflit entre vie privée et vie professionnelle (Hammer et alii, 2005). Nos résultats confortent en partie cette explication. Ils montrent, en effet, que le fait de bénéficier d'horaires flexibles diminue le temps de travail domestique des hommes cadres alors qu'il n'a aucun effet significatif sur ce 
celui des femmes cadres. Ainsi, par un sentiment de culpabilité (Berthe et alii, 2016), les femmes ne vont pas s'autoriser à utiliser les marges de manœuvre offertes par les horaires flexibles dans la gestion de leur agenda personnel pour s'accorder un temps supplémentaire à leurs loisirs. Ces résultats s'éclairent à lumière des recherches sur les effets, dans le partage des tâches domestiques entre hommes et femmes, de la réduction du temps de travail à $35 \mathrm{~h}$, à la fin des années 1990. Ainsi, Méda et Orain (2002, p. 30) notent que « le temps dégagé par la RTT n'a pas modifié l'aspect très fortement sexué des activités domestiques puisque $93 \%$ des femmes déclarent continuer à prendre en charge la plus grande partie de la lessive, $86 \%$ la plus grande part du repassage, environ $75 \%$ la plus grande part de la préparation des repas, du ménage et du rangement de la maison, $73 \%$ la plus grande part des courses alimentaires, alors que les hommes sont respectivement $74 \%$ et $51 \%$ à déclarer, depuis la RTT, prendre en charge l'essentiel du bricolage et du jardinage, donc des semi-loisirs ». Une autre explication à ces résultats inattendus rejoint les analyses de Kronberger et alii (2010). Les horaires flexibles, à l'inverse du but recherché, renforceraient les inégalités entre les hommes et les femmes en accentuant les obstacles à la progression des carrières féminines (Kornberger et alii, 2010). Les horaires flexibles peuvent se transformer en un stigmate, au sens de Goffman (1968), différenciant les salariés engagés dans leur travail de ceux qui ne le sont pas (Kornberger et alii, 2010). Ainsi, nos résultats montrent que le fait de bénéficier d'horaires à la carte diminue la perception que leurs perspectives de promotion soient satisfaisantes pour les femmes non-cadres. Ce dernier résultat converge avec la recherche de Premeaux et alii (2007) montrant que, dès lors que les conséquences perçues des horaires flexibles sur la carrière sont intégrées dans le modèle de régression, les horaires flexibles n'ont plus d'influence significative sur le conflit travail-famille. De même, dans le cadre d'une expérimentation, reproduisant une procédure de recrutement dans un cabinet d'audit comptable, Rogier et Padgett (2004) ont montré que les participants de sexes féminin et masculin avaient une perception biaisée des candidates à l'emploi, ayant bénéficié d'horaires de travail flexibles dans leurs expériences professionnelles précédentes. Les participants percevaient ces candidates comme étant moins attachées à leur travail, moins aptes à un avancement, moins ambitieuses et moins désireuses d'un avancement, et ce, malgré l'absence perçue de différences de compétences par rapport aux candidates n'ayant pas bénéficié d'horaires flexibles.

En outre, chez les hommes cadres, le bénéfice d'horaires libres et d'horaires à la carte augmente la perception de recevoir le respect et le mérite attendus, compte tenu du travail effectué. Ce n'est pas le cas des femmes cadres, selon nos résultats. Ainsi, sur la base de « suppositions sexuées »(Acker, 2006, p. 444), les directions d'entreprise semblent instrumentaliser les horaires flexibles dont bénéficient aux hommes cadres comme une forme de reconnaissance non monétaire en contrepartie des efforts fournis alors, qu'au mieux, elle n'a aucun effet sur la reconnaissance non monétaire des femmes cadres et, au pire, elle se transforme en un stigmate. Ces résultats convergent avec la notion «d'organisation genrée » proposée par Acker (1990). Cet auteur avance l'idée que la structure organisationnelle n'est pas neutre sur le plan du genre. Au contraire, les fiches de poste, la hiérarchie, les concepts utilisés pour penser l'organisation reposent implicitement sur un salarié désincarné et 
universel. C'est en fait un homme, selon Acker (1990), qui est subsumé au travers de cette image de salarié désincarné et universel.

Par ailleurs, dès lors que l'ensemble des effets pervers, associés à l'implantation d'horaires flexibles, sont intégrés dans notre modèle de régression, la relation négative entre la flexibilité des horaires -et plus précisément, le choix entre plusieurs horaires fixes- et la conciliation travail-famille, pour les femmes cadres, devient non-significative. C'est donc bien que les contreparties à l'implantation des horaires flexibles sont trop lourdes, pour les femmes salariées, pour permettre une réelle conciliation travail-famille. Dans le prolongement des réflexions d'Alber (2019), ces résultats renvoient aux injonctions paradoxales sous-jacentes au courant du High Performance Work System ${ }^{5}$ (HPWS) : l'autonomie concédée au salarié par l'employeur dans l'organisation de son temps de travail a pour contrepartie un contrôle indirect plus fort exercé via l'imposition d'une évaluation régulière, d'une direction par objectifs et d'une rémunération à la performance. Cette contrepartie peut être interprétée comme un moyen pour les employeurs de s'assurer que l'utilisation des horaires flexibles ne se fera pas au bénéfice d'une augmentation du temps alloué au temps libre. Elle présuppose une approche économique néoclassique du travail selon laquelle le travail est une désutilité définie par la renonciation au loisir consentie pour obtenir le salaire qui financera la consommation (Favereau, 2016).

\section{Conclusion}

Pour conclure, cette recherche laisse à penser que les horaires flexibles ne peuvent à eux-seuls réduire les inégalités professionnelles entre les hommes et les femmes. Pis, ils peuvent les accentuer, tout en n'améliorant pas le bien-être des femmes. Pour autant, il ne faudrait pas écarter tout recours aux horaires flexibles sur la base de ces recherches. Finalement, cette analyse quantitative renvoie implicitement aux manières dont les horaires flexibles sont mis en œuvre : ont-ils une contrepartie cachée, par exemple, en termes d'intensification du travail ou sont-ils réellement mis en œuvre dans la perspective de renforcer l'égalité professionnelle et le bien-être des salariés ? De la même manière, sont-ils considérés comme un stigmate différenciant les salariés fortement engagés des salariés désengagés ou sont-ils perçus par les managers comme un moyen de mieux équilibrer vie privée et vie professionnelle ? Par ailleurs, notre recherche interroge le sens et l'usage des horaires flexibles. N'est-ce qu'une autre forme de «flexibilité temporelle » (Everarere, 2012, p. 23), comme peut l'être le temps partiel dans la palette des différentes formes de flexibilité dont peut faire usage l'employeur, est-ce une flexibilité choisie ou, enfin, est-ce une « fausse alternative » (Maruani, 2017, p. 86) qui ne permet pas de distinguer ce qui relève du choix ou de la contrainte ? Notre recherche quantitative ne permet pas de répondre à ces questions. Il serait intéressant de la prolonger par

5 Ce courant du HPWS est nommé « néo-management » par Alber (2019). Un HPWS peut être considéré comme un ensemble cohérent de pratiques de gestion du travail (c'est-à-dire, selon Barreau (2005), englobant simultanément l'organisation du travail et la gestion des ressources humaines) à hautes performances (HPWP) créant des effets synergiques dans lesquels certaines pratiques se renforcent mutuellement pour augmenter l'efficacité et l'efficience organisationnelles (Posthuma et alii, 2013). 
le suivi d'une cohorte mixte de salariés combinant la passation de questionnaires et la réalisation d'entretiens, lors de plusieurs vagues, auprès de salariés et de praticiens.

\section{Bibliographie}

Acker, J. (1990), « Hierarchies, jobs, bodies: A theory of gendered organizations », Gender \& society, 4, p. 139-158.

Acker, J. (2006), « Inequality regimes: Gender, class, and race in organizations », Gender and Sociology, 20, p. 441-464.

Alber, A. (2019), "Tutoyer son chef. Entre rapports sociaux et logiques managériales », Sociologie $d u$ travail, 61, URL : http://journals.openedition.org/sdt/14517 ; DOI : 10.4000/sdt.14517.

Anderson, S. E., Coffey, B. S., Byerly, R. T. (2002). "Formal organizational initiatives and informal workplace practices: Links to work-family conflict and job-related outcomes".

Journal of management, 28, p. 787-810.

Ballesteros-Leiva, F., Poilpot-Rocaboy, G., St-Onge, S. (2016). "Relationships between lifedomain interactions and well-being: a comparison between men and women”. @ GRH, 2, p. 65-96.

Barreau, J. (2005), Gérer le travail, Rennes, PUR.

Batt, R., Monique, V. (2003). "Human Resources Practices as Predictors of Work-Family Outcomes and Employee Turnover". Industrial Relations, 42, p.189-220.

Berg, P., Appelbaum, E., Bailey, T., Kalleberg, A. L. (2004), “Contesting time: International comparisons of employee control of working time". ILR Review, 57, p. 331-349.

Berthe, B., Dumas, M., Poilpot-Rocaboy, G. (2016). " Le syndrome de la 'femme parfaite' ». Revue internationale de psychosociologie et de gestion des comportements organisationnels, 22, p. 187-206.

Bratberg, E., Dahl, S. A., and Risa, A. E. (2002). "The double burden'. Do combinations of career and family obligations increase sickness absence among women?". European Sociological Review, 18, p. 233-249.

Breaugh, J. A., Frye, N. K. (2008). Work-family conflict: The importance of family-friendly employment practices and family-supportive supervisors. Journal of business and psychology, 22, p. 345-353.

Brescoll, V. L., Glass, J. and Sedlovskaya, A. (2013). "Ask and ye shall receive? The dynamics of employer-provided flexible work options and the need for public policy". Journal of Social Issues, 69, p. 367-388.

Casper, W. J., Buffardi, L. C. (2004). "Work-life benefits and job pursuit intentions: The role of anticipated organizational support”. Journal of Vocational Behavior, 65, p. 391-410. 
Champagne, C., Pailhé, A., Solaz, A. (2015). Le temps domestique et parental des hommes et des femmes : quels facteurs d'évolutions en 25 ans ?, Économie et statistique, 478, p. 209-242.

Dodeler, N. L., Tremblay, D. G. (2014). Conciliation emploi-famille et horaires chez les paramédics (ambulanciers) : des différences selon l'âge et le genre. Management Avenir, 7, p. 171-187.

Dumas, M. (2008). Vie personnelle et vie professionnelle : vers un nouvel équilibre dans l'entreprise? Paris, Éditions EMS.

Everaere, C. (2012). Flexibilité appliquée aux ressources humaines. Compatibilités et contradictions. Revue française de gestion, 2, p. 13-32.

Favereau, O. (2016), Penser le travail pour penser l'entreprise, Paris, Presses des Mines

Felstead, A., Jewson, N. (2000). In Work, at Home: Towards an Understanding of Homeworking. New York/London: Routledge.

Goffman, E. (1968), Stigma: Notes on the management of spoiled identity, Harmondsworth, Penguin.

Greene, W.H. (2003), Econometric Analysis, Prentice Hall.

Hammer, L. B., Neal, M. B., Newsom, J. T., Brockwood, K. J., Colton, C. L. (2005), “A longitudinal study of the effects of dual-earner couples' utilization of family-friendly workplace supports on work and family outcomes". Journal of Applied Psychology, 90, p. 799-810.

Hofäcker, D., \& König, S. (2013). Flexibility and work-life conflict in times of crisis: a gender perspective. International Journal of Sociology and Social Policy, 33, p. 613-635.

Kelliher, C., Anderson, D. (2008). "For better or for worse? An analysis of how flexible working practices influence employees' perceptions of job quality". International Journal of Human Resource Management, 19, p. 419-431.

Kelly, E. L., Moen, P., Tranby, E. (2011). "Changing workplaces to reduce work-family conflict: Schedule control in a white-collar organization”. American Sociological Review, 76, p. 265-290.

Kergoat, D. (1978). « Ouvriers= ouvrières ? Propositions pour une articulation théorique de deux variables : sexe et classe sociale ». Critiques de l'économie politique, 5, p. 65-97.

Kerkhofs, M., Chung, H., \& Ester, P. (2008). Working time flexibility across Europe: a typology using firmâ level data. Industrial Relations Journal, 39(6), 569-585.

Kornberger, M., Carter, C., Ross-Smith, A. (2010), « Changing gender domination in a Big Four accounting firm: Flexibility, performance and client service in practice », Accounting, Organizations and Society, 35, p. 775-791. 
Lapeyre, N., Le Feuvre, N. (2004). Concilier l'inconciliable ? Le rapport des femmes à la notion de « conciliation travail-famille» dans les professions libérales en France. Nouvelles questions féministes, 23, 42-58.

Lapierre, L. M., Allen, T. D. (2006). « Work-supportive family, family-supportive supervision, use of organizational benefits, and problem-focused coping: implications for work-family conflict and employee well-being". Journal of Occupational Health Psychology, 11, p. 169-181.

Lott, Y. (2018). "Does flexibility help employees switch off from work? Flexible workingtime arrangements and cognitive work-to-home spillover for women and men in Germany". Social Indicators Research, p. 1-24.

Lott, Y., \& Chung, H. (2016). "Gender discrepancies in the outcomes of schedule control on overtime hours and income in Germany”. European Sociological Review, 32, p. 752-765.

Maruani, M. (2017), Travail et emploi des femmes, Paris, La Découverte.

Méda, D., Orain, R. (2002). « Transformations du travail et du hors travail : le jugement des salaries sur la réduction du temps de travail ». Travail et Emploi, 90, p. 23-38.

Mbengue, A., Vandangeon-Derumez, I. (2003), « Analyse causale et modélisation », in Thiétart, R.A. (coord.), Méthodes de recherche en management, Paris, Dunod, p. 335-372.

Moen, P., Yu, Y. (2000). "Effective work/life strategies: working couples, work conditions, gender, and life quality". Social Problems, 47, p. 291-326.

Pierce, J. L., Newstrom, J. W. (1980). "Toward a conceptual clarification of employee responses to flexible working hours: A work adjustment approach". Journal of Management, 6, p. 117-134.

Pigeyre, F. (1999), « Vers une gestion des ressources humaines sexuée ? Le cas des carrières des femmes cadres », Revue Française de Gestion, 126, p. 47-55.

Pigeyre, F. (2001), «Femmes dirigeantes : les chemins du pouvoir », in Bouffartigue, P. Cadres : la grande rupture, La Découverte, p. 269-280.

Posthuma, R. A., Campion, M. C., Masimova, M., \& Campion, M. A. (2013). “A high performance work practices taxonomy: Integrating the literature and directing future research". Journal of Management, 39, p. 1184-1220.

Premeaux, S. F., Adkins, C. L., \& Mossholder, K. W. (2007). Balancing work and family: a field study of multiâdimensional, multitrole workw family conflict. Journal of Organizational Behavior, 28, p. 705-727.

Rogier SA, Padgett MY (2004). The Impact of Utilizing a Flexible Work Schedule on the Perceived Career Advancement Potential of Women. Human Resource Development Quarterly 15, p. 89-106. 
Schieman, S., Melissa, M., and Paul, G. (2009). "When Work Interferes with Life: WorkNonwork Interference and the Influence of Work-Related Demands and Resources".

American Sociological Review, 74, p. 966-988.

Thomas, L. T., Ganster, D. C. (1995). "Impact of family-supportive work variables on workfamily conflict and strain: A control perspective”. Journal of Applied Psychology, 80, p. 6-15.

Thompson, C. A., Beauvais, L. L., Lyness, K. S. (1999). «When work-family benefits are not enough: The influence of work-family culture on benefit utilization, organizational attachment, and work-family conflict". Journal of Vocational Behavior, 54, p. 392-415.

Tremblay, D. G., d'Amours, L. (2016). Conciliation travail-famille, encore et toujours un défi. Gestion, 41(1), 22-25.

Williams, J. C., Blair-Loy, M. Berdahl, J. L. (2013). “Cultural schemas, social class, and the flexibility stigma”. Journal of Social Issues, 69, p. 209-234. 